\title{
Neuropatia autonômica no diabetes: a importância de uma complicação pouco investigada
}

\author{
Lucianne R. M. Tannus ${ }^{1 *}$
}

\begin{abstract}
Resumo
A neuropatia autonômica cardiovascular (NAC), apesar de ser considerada uma complicação crônica comum no paciente com diabetes mellitus (DM) e associada com o aumento de morbimortalidade cardiovascular (CV), ainda permanece subdiagnosticada. A presente revisão tem o objetivo de descrever os critérios diagnósticos da NAC, os fatores clínicolaboratoriais relacionados com a sua presença e as estratégias terapêuticas disponíveis. A NAC está presente em $25 \%$ dos pacientes com DM tipo1(DM1) e em 34\% dos pacientes com DM tipo 2(DM2). Encontra-se associada à neuropatia periférica, assim como à presença de hipertensão arterial sistêmica (HAS), dislipidemia, nefropatia, retinopatia diabética, doença vascular periférica (DVP) e os principais fatores de risco são idade, duração do DM e presença de controle glicêmico inadequado. A NAC pode se manifestar através de taquicardia de repouso, hipotensão postural, intolerância à atividade física, isquemia miocárdica silenciosa, aumento do intervalo QT, cardiomiopatia diabética e ausência de descenso noturno de pressão arterial sistólica (PAS). Os sinais e sintomas associados à presença da NAC surgem tardiamente na evolução da doença, tornando necessária a utilização de testes reflexos $\mathrm{CV}$ para identificação precoce da NAC (teste da respiração profunda, teste de Valsalva, teste do ortostatismo e pesquisa de hipotensão ortostática). De acordo com as recomendações atuais, é necessária a presença de pelo menos dois testes reflexos $\mathrm{CV}$ alterados para o diagnóstico definitivo de NAC. As estratégias terapêuticas disponíveis são tratamento dos fatores de RCV (HAS, dislipidemia e diabetes), além da prática de exercícios físicos, uso de betabloqueadores, inibidores da enzima conversora de angiotensina (iECA) e ácido alfalipoico. A NAC ainda permanece subdiagnosticada. O rastreamento é recomendado para o diagnóstico de pacientes com DM2 e após cinco anos do diagnóstico de pacientes com DM1 para permitir o diagnóstico precoce tratamento adequado e prevenção e reversão das alterações autonômicas de CV.
\end{abstract}

Descritores: Diabetes mellitus; Risco; Doenças cardiovasculares; Neuropatias diabéticas; Angiopatias diabéticas.

\begin{abstract}
Autonomic neuropathy in diabetes: the importance of a complication poorly investigated

Cardiovascular autonomic neuropathy (CAN) despite being considered a common chronic complication in patients with diabetes mellitus (DM) and associated with increased morbidity and mortality from cardiovascular (CV) disease remains underdiagnosed. This review aims to describe the diagnostic criteria of CAN, clinical and laboratory factors associated with
\end{abstract}

1. Disciplina de Diabetes. Departamento de Medicina Interna. Faculdade de Ciências Médicas. Universidade do Estado do Rio de Janeiro. Rio de Janeiro, RJ, Brasil.

\author{
*Endereço para correspondência: \\ HUPE, UERJ \\ Boulevard 28 de Setembro, 77 \\ Rio de Janeiro, RJ, Brasil. CEP: 20551-030. \\ E-mail: luciannetannus@ig.com.br
}

Revista HUPE, Rio de Janeiro, 2015;14(4):64-69

doi: 10.12957/rhupe.2015.20060

Recebido em 28/11/2014. Aprovado em 08/12/2015.

CAN and the available therapeutic strategies. CAN is present in $25 \%$ of patients with type $1 \mathrm{DM}$ (T1DM) and in $34 \%$ of patients with type $2 \mathrm{DM}$ (T2DM). It is associated with peripheral neuropathy as well as the presence of hypertension, dyslipidemia, nephropathy, retinopathy, peripheral artery disease and the risk factors are age, diabetes duration, and presence of inadequate glycemic control. The following clinical manifestations are associated with CAN: resting tachycardia, postural hypotension, impaired physical activity, silent myocardial ischemia, increased QT interval, diabetic cardiomyopathy and absence of nocturnal systolic blood pressure decrease. The signs and symptoms associated with CAN appear late in the course of the disease and, therefore the use of the following CV reflex tests are essential for early identification of CAN (deep breathing test, Valsalva test, the standing position, making it necessary research and orthostatic hypotension). According to current recommendations, the presence of at least two CV altered reflexes tests is required for definitive diagnosis of CAN. The available treatment for the management of CAN are the treatment of CV risk factors (hypertension, dyslipidemia and diabetes), besides physical exercise, use of beta blockers, inhibitors of angiotensin-converting enzyme (ACE) and alpha lipoic acid. CAN remains underdiagnosed. Screening should be performed at diagnosis of patients with T2DM and after 5 years of diagnosis in patients with T1DM to allow early diagnosis, appropriate treatment and prevention or reversal of $\mathrm{CV}$ autonomic alterations.

Keywords: Diabetes mellitus; Risk; Cardiovascular diseases; Neuropatias diabéticas; Diabetic angiopathies..

\section{Resumen}

Neuropatía autonómica en la diabetes: la importancia de una complicación poco investigada 
Neuropatía autonómica cardiovascular (NAC), a pesar de considerarse una complicación crónica común en el paciente con diabetes mellitus (DM), y asociada con el aumento de la morbimortalidad cardiovascular (CV), todavía es subdiagnosticada Esta revisión tiene el objetivo de describir los criterios de diagnóstico de la NAC, los factores clínicos y de laboratorio relacionados con su presencia y las estrategias terapéuticas disponibles. La NAC está presente en el 25\% de los pacientes con DM tipo 1(DM1) y en el 34\% de los pacientes con DM tipo 2 (DM2). Está asociada con la neuropatía periférica, así como con la presencia de hipertensión arterial sistémica (HAS), dislipidemia, nefropatía, retinopatía diabética, enfermedad vascular periférica (EVP) y los principales factores de riesgo: la edad, la duración de la DM y la presencia de control glucémico inadecuado. La NAC puede manifestarse a través de la taquicardia de reposo, hipotensión postural, intolerancia a la actividad física, isquemia miocárdica silenciosa, aumento del intervalo QT, cardiomiopatía diabética y ausencia del descenso nocturno de la presión arterial sistólica (PAS). Las señales y síntomas asociados con la presencia de NAC surgen

\section{Introdução}

A neuropatia autonômica cardiovascular (NAC), apesar de ser considerada uma complicação crônica comum $^{1}$ no paciente com diabetes mellitus (DM) e associada com o aumento de morbimortalidade cardiovascular, ainda permanece subdiagnosticada. ${ }^{1,2}$ Os sinais e sintomas associados à presença da NAC surgem tardiamente na evolução da doença, tornando necessária a utilização de testes reflexos cardiovasculares para identificação precoce da NAC.-5

A doença cardiovascular (DCV) representa a principal causa de morte em pacientes com DM. As taxas de mortalidade por DCV em pacientes com DM tipo 2 (DM2) e DM tipo1(DM1) são, respectivamente, 2-4 vezes e 10 vezes maior do que em indivíduos saudáveis sem DM. A incidência de doença arterial coronariana (DAC) é aproximadamente $1 \%$ a $\%$ ao ano entre jovens assintomáticos com DM1; ;-8 os mesmos apresentam maiores taxas de mortalidade por DAC e doença cerebrovascular quando comparados à população geral. ${ }^{6-8}$

O excesso de mortalidade em DM1 parece não ser totalmente justificado pela presença dos clássicos fatores de risco cardiovascular (RCV), tais como hipertensão arterial sistêmica (HAS) e dislipidemia e a presença das complicações microvasculares parece contribuir para o aumento nas taxas de mortalidade. ${ }^{9}$ Um estudo finlandês avaliou a incidência de DCV em 5.148 pacientes com DM1 e se observou que a presença de nefropatia diabética aumentou em dez vezes o risco de DAC. ${ }^{9}$

O sistema nervoso autonômico (SNA), por ser res- tardíamente en la evolución de la enfermedad, haciéndose necesario el uso de pruebas de reflejos CV para la identificación temprana de la NAC (prueba de respiración profunda, prueba de Valsalva, prueba de Ortostatismo e investigación de hipotensión ortostática). Según las recomendaciones actuales, se requiere la presencia de al menos dos pruebas de reflejos $\mathrm{CV}$ alterados para el diagnóstico definitivo de la NAC. Las estrategias terapéuticas disponibles: tratamiento de los factores de riesgo cardiovascular (hipertensión, dislipidemia y diabetes), además de la práctica de ejercicios físicos, uso de betabloqueadores, inhibidores de la enzima convertidora de la angiotensina (iECA) y ácido alfa-lipoico. La NAC todavía es subdiagnosticada. Se recomienda el rastreo para el diagnóstico de pacientes con DM2 y después de cinco años el diagnóstico de pacientes con DM1, para permitir el diagnóstico precoz, tratamiento adecuado, prevención y reversión de las alteraciones autonómicas de $\mathrm{CV}$.

Palabras clave: Diabetes mellitus; Riesgo; Enfermedades cardiovasculares; Neuropatías diabéticas; Angiopatías diabéticas.

ponsável pela regulação da frequência cardíaca (FC) e pelo tônus vascular, pode contribuir para o surgimento de rigidez arterial, condição reconhecida como preditora de risco e mortalidade cardiovascular em DM. ${ }^{10}$ Estudos anteriores já demonstraram que a NAC [determinada por alterações da razão expiração: inspiração (E:I)] apresenta correlação com rigidez arterial e com redução da estimativa de perfusão miocárdica em DM1. NAC também foi associada com hipertrofia ventricular esquerda e disfunção diastólica nesse grupo de pacientes. A rigidez arterial parece contribuir para a disfunção diastólica ventricular esquerda e esta alteração vascular poderia ser a ligação entre NAC e DCV em DM. ${ }^{10}$

Além disso, estudo realizado por Quattrini e colaboradores ${ }^{11}$ demonstrou que pacientes com grave dor neuropática decorrente de neurite insulínica apresentam anormalidades na vascularização neural (aumento do shunt epineural com consequente redução do fluxo endoneural). Estes dados revelam uma ligação entre neuropatia diabética e alterações vasculares, e foram ratificados por outros estudos que evidenciaram significativa melhora da dor neuropática através do uso de vasodilatadores..$^{12} \mathrm{~A}$ interrelação entre neuropatia diabética e disfunção vascular também pode estar associada com o estresse oxidativo, o qual promove um desbalanço entre fatores vasodilatadores e vasoconstrictores endoteliais, determinando redução de óxido nítrico, prostaciclina, fator hiperpolarizante derivado do endotélio, aumento de angiotensina-II e endotelina, culminando com dano vascular endotelial. ${ }^{12}$ 


\section{Artigo de revisão}

A NAC tem sido apontada como uma das possíveis causas de morte súbita e representa um forte indicador de RCV, tanto em DM1 quanto em DM2. Pacientes diabéticos com NAC apresentam taxas de mortalidade de 16 a 53\% em cinco anos, dependendo da gravidade da NAC. ${ }^{13}$ No estudo Detection of silent myocardial ischemia in asymptomatic diabetic subjects (DIAD), realizado em pacientes com DM2, a presença de NAC, determinada pela redução da razão de Valsalva, foi preditora de isquemia miocárdica silenciosa, independente da presença dos tradicionais fatores de RCV, tais como HAS, idade, sexo e tabagismo. ${ }^{14}$ No estudo European Epidemiology and Prevention of Diabetes (EURODIAB), a NAC foi identificada em $1 / 3$ de pacientes com DM1 e foi associada com outros fatores de risco cardiovascular após ajuste para idade, níveis de hemoglobina glicada (HbA1c) e duração do diabetes. ${ }^{15}$ Dados do estudo Action to Control Cardiovascular Risk in Diabetes (ACCORD) demonstraram que pacientes com NAC apresentaram taxa de mortalidade cerca de 1,55-2,14 vezes maiores do que pacientes sem NAC. ${ }^{16}$

\section{Objetivo}

A presente revisão tem o objetivo de descrever os critérios diagnósticos da NAC, os fatores clínicolaboratoriais relacionados com a sua presença e as estratégias terapêuticas disponíveis.

\section{Epidemiologia}

A neuropatia autonômica cardiovascular (NAC) pode estar presente desde o diagnóstico do DM e, frequentemente, é subdiagnosticada. Sua prevalência varia de $2,6 \%$ a 90\% entre os pacientes com DM, ${ }^{13}$ dependendo do método diagnóstico empregado, sendo encontrada em cerca de $25 \%$ e $34 \%$ dos pacientes com DM1 e DM2, respectivamente. ${ }^{2}$ Pode estar presente ao diagnóstico em 7\% dos casos de DM e a incidência anual é de cerca de 6\% e 2\% em pacientes com DM2 e DM1, respectivamente. ${ }^{17}$ A incidência de NAC aumenta com a idade, duração do diabetes e presença de controle glicêmico inadequado. ${ }^{18}$ Em geral, encontra-se associada à neuropatia periférica sensitivo-motora de membros inferiores (em até 62,5\% dos casos), assim como à presença de outros fatores de RCV, como HAS, dislipidemia, nefropatia e retinopatia diabéticas, além de doença vascular periférica (DVP). ${ }^{18}$

Existem diferenças em relação aos aspectos clínicos da NAC em pacientes com DM1 e DM2. Os sinais e sintomas da NAC são mais precoces e mais prevalentes em pacientes com DM2, estão associados com maior tempo de exposição à hiperglicemia, enquanto que em pacientes com DM1 os sintomas são mais tardios e quando aparecem, em geral, estão associados à gravidade de doença. ${ }^{19}$ Em pacientes com DM1, NAC está associada com a presença de albuminúria, retinopatia e neuropatia periférica, enquanto que em pacientes com DM2, NAC parece estar associada com hipertensão arterial sistêmica (HAS), índice de massa corporal (IMC), tabagismo, duração de DM e níveis de HbA1c, sugerindo associação de NAC com complicações microvasculares e macrovasculares em pacientes com DM1 e DM2, respectivamente. ${ }^{19}$

\section{Fisiopatologia}

A fisiopatologia da NAC é multifatorial e envolve vários mecanismos que resultam em dano neuronal. $\mathrm{O}$ aumento dos níveis intracelulares de glicose leva à ativação de diversas vias envolvidas no desenvolvimento das complicações micro e macrovasculares do DM: a via dos polióis, via da hexosamina, formação de produtos finais de glicação avançada (AGE) e a ativação da proteína quinase $\mathrm{C}$ (PKC). ${ }^{20}$ Todas estas vias apresentam um denominador comum: o aumento do estresse oxidativo que resulta de um desequilíbrio entre a produção de espécies reativas de oxigênio (EROS) e as defesas antioxidantes..$^{20,21}$ As EROS são moléculas quimicamente instáveis e altamente reativas inicialmente formadas pela produção de $\mathrm{O}_{2}$-mitocondrial induzido pela hiperglicemia, causando dano a estruturas celulares, alterações na expressão genética, fatores de transcrição além de alterações microvasculares. ${ }^{22}$

Existem evidências recentes de que a inflamação seja o mecanismo-chave para o desenvolvimento de complicações crônicas do DM e que a resposta inflamatória seja controlada, em parte, pelo circuito do sistema nervoso autonômico (SNA). ${ }^{1,23}$ É descrito que exista um constante estado inflamatório de baixo grau em pacientes com DM levando ao estímulo constante de vias aferentes do SNA (nervo vago) por citoquinas, particularmente IL-1. Os sinais aferentes vagais são transmitidos até o núcleo do trato solitário que vai estimular vias eferentes dos SNS até o gânglio celíaco, com consequente estimulação do nervo esplênico e ativação da cascata inflamatória nos macrófagos esplênicos. Simultaneamente, ocorre o estímulo de vias eferentes colinérgicas do SNPS (representado pelo nervo vago) que produzem inibição da resposta imune inata e supressão da inflamação. ${ }^{1}$ Desta forma, parece ocorrer uma imunomodulação mediada peloSNA [estímulo induzido pelo sistema nervoso simpático(SNS) e inibição 
induzida pelo sistema nervoso parassimpático(SNPS)].1

O desequilíbrio simpatovagal com redução da atividade do SNPS e hiperatividade simpática pode iniciar a cascata inflamatória, estabelecendo um link entre hiperglicemia, inflamação, estresse oxidativo e disfunção autonômica. ${ }^{1,23}$

\section{Diagnóstico}

Caracteristicamente, a NAC pode se manifestar através de taquicardia de repouso, hipotensão postural, intolerância à atividade física (consequente a bloqueio cronotrópico e inotrópico cardíacos), regulação vasomotora coronariana anormal (com redução do limiar para isquemia), ${ }^{2}$ aumento do intervalo QT (com risco de arritmias e morte súbita), alteração das funções sistólica e diastólica do ventrículo esquerdo (cardiomiopatia diabética), redução de resposta respiratória à hipóxia (aumentando o risco de complicações perioperatórias), variação circadiana anormal do sistema simpatovagal [ausência de descenso noturno de pressão arterial sistólica (PAS)], além de maior risco para isquemia miocárdica silenciosa. ${ }^{2}$

A alteração do balanço simpatovagal reduz a resposta de FC e pressão arterial (PA) e limita o aumento do débito cardíaco durante o exercício físico, ocasionando intolerância ao exercício. Portanto, pacientes com NAC devem ser submetidos à investigação de DAC antes de iniciar um programa de exercícios físicos. ${ }^{2,17,18}$

Hipotensão ortostática $(\mathrm{HO})$ pode estar presente em 6-32\% dos pacientes com NAC dependendo do critério diagnóstico utilizado (20 ou $30 \mathrm{mmHg}$ ). Sintomas de hipotensão postural (tonturas, escotomas cintilantes, turvação visual, sensação de desmaio) podem estar presentes em 4-18\% dos pacientes com NAC, são mais intensos pela manhã, nos períodos pós-prandiais, em ambientes com temperaturas elevadas, durante atividade física e ortostatismo prolongado, ${ }^{17}$ e podem ser precipitados por algumas medicações, tais como vasodilatadores, diuréticos, antidepressivos tricíclicos e insulina. ${ }^{17}$

De acordo com a Associação Americana de Diabetes (ADA), o rastreamento de NAC deve ser realizado ao diagnóstico em todos os pacientes com DM2 eem todos os pacientes com DM1 após cinco anos do diagnóstico. ${ }^{24}$

Os testes reflexos cardiovasculares avaliam as oscilações da FC em resposta aos testes de respiração profunda, teste de Valsalva e ortostatismo e as variações de PA durante o ortostatismo e são considerados os testes padrão-ouro para avaliação da função autonômica cardiovascular, segundo consenso da Conferência de
Neuropatia Diabética realizado em San Antonio em 1992, ${ }^{3}$ da Neuropatia Diabética de Toronto ${ }^{4,17}$ e do Grupo de Estudos de Neuropatia Diabética da Sociedade Italina de Diabetologia.

A análise espectral dos intervalos RR (análise do domínio da frequência) durante repouso de cinco minutos permite a determinação de parâmetros de domínio de tempo e de frequência influenciadas pelo SNS e SNPS:2,5 1) componente de frequências muito baixas (FMB) $(0,01$ a $0,04 \mathrm{~Hz})$, que está relacionado com as flutuações do tônus vasomotor ligadas à termorregulação e sudorese (controle simpático), componentes de frequências baixas (FB) $(0,04 \mathrm{a}$ 0,15 Hz) associado ao reflexo barorreceptor (controle simpático com modulação vagal) e componentes de frequências altas (FA) $(0,15$ a $0,5 \mathrm{~Hz}$ ), relacionado com o controle parassimpático (nervo vago)., ${ }^{1,2}$ Os parâmetros de VFC obtidos parecem ser mais sensíveis e as alterações da VFC mais precoces do que os testes reflexos cardiovasculares e, apesar de representarem testes de baixo custo, não invasivos, de fácil realização e não necessitar de cooperação ativa dos pacientes, apresentam baixa reprodutibilidade, ${ }^{25}$ sendo, portanto, considerados como testes adicionais para o diagnóstico de NAC.-5

O diagnóstico de NAC é estabelecido com base em alterações dos testes reflexos cardiovasculares (teste da respiração profunda, teste de Valsalva, teste do ortostatismo e pesquisa de hipotensão ortostática) e, de acordo com as recomendações atuais, ${ }^{5}$ é necessária a presença de pelo menos dois testes reflexos cardiovasculares alterados para o diagnóstico de NAC definida, enquanto pacientes com alteração de apenas um teste reflexo cardiovascular foram classificados como NAC incipiente.

\section{Tratamento}

Vários estudos vêm relatando que o controle glicêmico intensivo pode retardar o aparecimento e reverter algumas alterações da NAC. Dados do estudo Steno $2^{26}$ demonstraram redução de $68 \%$ no risco de progressão da NAC em pacientes com DM2 e albuminúria submetidos ao tratamento intensivo multifatorial (dieta, exercício físico, interrupção do tabagismo, além de controle da PA, dislipidemia e hiperglicemia ${ }^{26}$ enquanto que redução de 53\% da prevalência da NAC foi observada no estudo The Diabetes Control and Complications Trial (DCCT) após 6,5 anos de acompanhamento de pacientes com DM1 submetidos à insulinoterapia intensiva e estes efeitos benéficos persistiram após 13-14 anos do término do estudo. ${ }^{27}$

O estudo Diabetes Prevention Program (DPP) ${ }^{28}$ 


\section{Artigo de revisão}

demonstrou que modificações do estilo de vida foram superiores à metformina e placebo na melhora dos índices de VFC (variabilidade de frequência cardíaca), e estudos recentes demonstraram que o exercídico físico foi capaz de induzir melhora dos parâmetros de VFC tanto em pacientes com DM1 quanto DM2. ${ }^{29}$

O uso do antioxidante ácido alfalipoico demonstrou melhora dos parâmetros de VFC em pacientes com DM2 durante um período de quatro meses. ${ }^{30}$

Alguns estudos observaram melhora dos parâmetros de VFC com o uso de inibidores dos sistema renina angiotensina aldosterona (SRAA) e betabloqueadores. ${ }^{22}$

O tratamento da hipotensão ortostática ainda representa um desafio na prática clínica. Medidas não farmacológicas incluem aumento do consumo de líquidos, uso de meias elásticas, evitar rápidas mudanças posturais e medicamentos agravantes da hipotensão (antidepressivos tricíclicos), realização de refeições pequenas e frequentes, além de evitar exercícios físicos que induzam a realização de manobra de Valsalva. ${ }^{22}$

Medidas farmacológicas devem ser indicadas quando as estratégias não farmacológicas falham no tratamento da hipotensão postural mas apresentam dificuldades pelo risco de indução de hipertensão supina. Duas estratégias de primeira linha estão disponíveis para tratamento da hipotensão postural: a 9- $\alpha$ fludrocortisona, um mineralcorticoide sintético (doses 100-400 $\mu$ /dia) que induz retenção de sódio e expansão plasmática e a midodrina, agonista adrenérgico seletivo periférico, que promove vasoconstricção periférica de veias e arteríolas. ${ }^{22}$

\section{Conclusões}

NACé uma complicação crônica comum que afeta cerca de 30\% dos pacientes com DM, mas permanece subdiagnosticada. Os testes reflexos cardiovasculares constituem os testes padrão-ouro para o diagnóstico de NAC e pelo menos dois testes devem estar alterados para o diagnóstico definitivo de NAC. Os principais fatores de risco são idade, duração do DM, HbA1c, HAS, FC de repouso, a presença de nefropatia, neuropatia e retinopatia diabética. Exercício físico associado com controle glicêmico intensivo, além de uso de algumas medicações, como ácido alfalipoico, $\beta$-bloqueadores e inidores do SRAA estão dentre as estratégias terapêuticas disponíveis para prevenção/reversão das alterações do SNA. O rastreamento da NAC deve fazer parte da investigação de complicações microvasculares do DM para redução de morbimortalidade cardiovascular.

\section{Referências}

1. Vinik Al, Erbas T, Casellini CM. Diabetic cardiac autonomic neuropathy, inflammation and cardiovascular disease. J Diabetes Investig. 2013 Jan;4(1):4-18.

2. Rolim LC, Sa JR, Chacra AR, et al. Diabetic cardiovascular autonomic neuropathy: risk factors, clinical impact and early diagnosis. Arq Bras Cardiol. 2008 Apr;90(4):e24-31.

3. Consensus statement: Report and recommendations of the San Antonio conference on diabetic neuropathy. American Diabetes Association American Academy of Neurology. Diabetes Care. 1988 Jul-Aug;11(7):592-7.

4. Tesfaye S, Boulton AJ, Dyck PJ, et al. Diabetic neuropathies: update on definitions, diagnostic criteria, estimation of severity, and treatments. Diabetes Care. 2010 Oct;33(10):2285-93.

5. Spallone V, Bellavere F, Scionti L, et al. Recommendations for the use of cardiovascular tests in diagnosing diabetic autonomic neuropathy. Nutr Metab Cardiovasc Dis. 2011 Jan;21(1):69-78.

6. Libby P, Nathan DM, Abraham K, et al. Report of the National Heart, Lung, and Blood Institute-National Institute of Diabetes and Digestive and Kidney Diseases Working Group on Cardiovascular Complications of Type 1 Diabetes Mellitus. Circulation. 2005 Jun 28;111(25):3489-93.

7. Zgibor JC, Piatt GA, Ruppert K, et al. Deficiencies of cardiovascular risk prediction models for type 1 diabetes. Diabetes Care. 2006 Aug;29(8):1860-5.

8. Laing SP, Swerdlow AJ, Slater SD, et al. Mortality from heart disease in a cohort of 23,000 patients with insulin-treated diabetes. Diabetologia. 2003 Jun;46(6):760-5.

9. Tuomilehto J, Borch-Johnsen K, Molarius A, et al. Incidence of cardiovascular disease in Type 1 (insulin-dependent) diabetic subjects with and without diabetic nephropathy in Finland. Diabetologia. 1998 Jul;41(7):784-90.

10. Prince CT, Secrest AM, Mackey RH, et al Cardiovascular autonomic neuropathy, HDL cholesterol, and smoking correlate with arterial stiffness markers determined 18 years later in type 1 diabetes. Diabetes Care. 2010 Mar;33(3):652-7.

11. Quattrini C, Harris ND, Malik RA, et al. Impaired skin microvascular reactivity in painful diabetic neuropathy. Diabetes Care. 2007 Mar;30(3):655-9.

12. Tesfaye S, Malik R, Harris N, et al. Arterio-venous shunting and proliferating new vessels in acute painful neuropathy of rapid glycaemic control (insulin neuritis). Diabetologia. 1996 Mar;39(3):329-35.

13. Schmid H. [Cardiovascular impact of the autonomic neuropathy of diabetes mellitus]. Arq Bras Endocrinol Metabol. 2007 Mar;51(2):232-43.

14. Wackers FJ, Young LH, Inzucchi SE, et al. Detection of silent myocardial ischemia in asymptomatic diabetic subjects: the DIAD study. Diabetes Care. 2004 Aug;27(8):1954-61.

15. Kempler P, Tesfaye S, Chaturvedi N, et al. Autonomic neuropathy is associated with increased cardiovascular risk factors: the EURODIAB IDDM Complications Study. Diabet Med. 2002 Nov; 19(11):900-9.

16. Pop-Busui R, Evans GW, Gerstein HC, et al. Effects of cardiac autonomic dysfunction on mortality risk in the Action to Control Cardiovascular Risk in Diabetes (ACCORD) trial. Diabetes Care. 2010 Jul;33(7):1578-84. 
Lucianne R. M. Tannus • Neuropatia autonômica no diabetes: a importância de uma complicação pouco investigada

17. Spallone V, Ziegler D, Freeman R, et al. Cardiovascular autonomic neuropathy in diabetes: clinical impact, assessment, diagnosis, and management. Diabetes Metab Res Rev. 2011 Oct;27(7):639-53.

18. Vinik AI, Ziegler D. Diabetic cardiovascular autonomic neuropathy. Circulation. 2007 Jan 23;115(3):387-97.

19. Rolim LC, Sa JR, Chacra AR, et al. [Clinical heterogeneity and coexistence of diabetic neuropathies: difference and similarities between types 1 and 2 diabetes mellitus]. Arq Bras Endocrinol Metabol. 2009 Oct;53(7):818-24.

20. Berry C, Brosnan MJ, Fennell J, et al. Oxidative stress and vascular damage in hypertension. Curr Opin Nephrol Hypertens. 2001 Mar;10(2):247-55.

21. Bandeira de SM, da Fonseca LJ, Guedes G da S, et al. Oxidative stress as an underlying contributor in the development of chronic complications in diabetes mellitus. Int $\mathrm{J}$ Mol Sci. 2013;14(2):3265-84.

22. Dimitropoulos G, Tahrani AA, Stevens MJ. Cardiac autonomic neuropathy in patients with diabetes mellitus. World J Diabetes. 2014 Feb 15;5(1):17-39.

23. Vinik Al, Maser RE, Ziegler D. Autonomic imbalance: prophet of doom or scope for hope? Diabet Med. 2011 Jun;28(6):643-51.

24. Standards of medical care in diabetes--2013. Diabetes Care. 2013 Jan;36 Suppl 1:S11-66.
25. Tannus LR, Sperandei S, Montenegro Jr RM, et al. Reproducibility of methods used for the assessment of autonomous nervous system's function. Auton Neurosci. 2013 Oct;177(2):275-9.

26. Gaede P, Vedel P, Larsen N, et al. Multifactorial intervention and cardiovascular disease in patients with type 2 diabetes. N Engl J Med. 2003 Jan 30;348(5):383-93.

27. The effect of intensive diabetes therapy on measures of autonomic nervous system function in the Diabetes Control and Complications Trial (DCCT). Diabetologia. 1998 Apr;41(4):416-23.

28. Carnethon MR, Prineas RJ, Temprosa M, et al. The association among autonomic nervous system function, incident diabetes, and intervention arm in the Diabetes Prevention Program. Diabetes Care. 2006 Apr;29(4):914-9. 29. Voulgari C, Pagoni S, Vinik A, et al. Exercise improves cardiac autonomic function in obesity and diabetes. Metabolism. 2013 May;62(5):609-21.

30. Ziegler D, Schatz H, Conrad F, et al. Effects of treatment with the antioxidant alpha-lipoic acid on cardiac autonomic neuropathy in NIDDM patients. A 4-month randomized controlled multicenter trial (DEKAN Study). Deutsche Kardiale Autonome Neuropathie. Diabetes Care. 1997 Mar;20(3):369-73. 